\title{
NASA SOIL MOISTURE ACTIVE PASSIVE MISSION STATUS AND SCIENCE PERFORMANCE
}

\author{
Simon Yueh ${ }^{1}$, Dara Entekhabi ${ }^{2}$, Peggy O’Neill ${ }^{3}$, Eni Njoku ${ }^{1}$, and Jared Entin ${ }^{4}$ \\ ${ }^{1}$ Jet Propulsion Laboratory, California Institute of Technology, CA, USA \\ ${ }^{2}$ Massachusetts Institute of Technology, Massachusetts, USA \\ ${ }^{3}$ Goddard Space Flight Center, National Aeronautics and Space Administration, Maryland, USA \\ ${ }^{4}$ National Aeronautics and Space Administration Headquarters, DC, USA
}

\begin{abstract}
The Soil Moisture Active Passive (SMAP) observatory was launched January 31, 2015, and its L-band radiometer and radar instruments became operational since mid-April 2015. The SMAP radiometer has been operating flawlessly, but the radar transmitter ceased operation on July 7 . This paper provides a status summary of the calibration and validation of the SMAP instruments and the quality assessment of its soil moisture and freeze/thaw products. Since the loss of the radar in July, the SMAP project has been conducting two parallel activities to enhance the resolution of soil moisture products. One of them explores the Backus Gilbert optimum interpolation and de-convolution techniques based on the oversampling characteristics of the SMAP radiometer. The other investigates the disaggregation of the SMAP radiometer data using the European Space Agency's Sentinel-1 C-band synthetic radar data to obtain soil moisture products at about 1 to $3 \mathrm{~km}$ resolution. In addition, SMAP's L-band data have found many new applications, including vegetation opacity, ocean surface salinity and hurricane ocean surface wind mapping. Highlights of these new applications will be provided.
\end{abstract}

Index Terms - Soil moisture, radiometer, radar, microwave remote sensing

\section{INTRODUCTION}

Soil moisture is a primary state variable of hydrology and the water cycle over land. In diverse Earth and environmental science disciplines, this state variable is either an initial condition or a boundary condition of relevant hydrologic models. Applications such as weather forecasting, and skillful modeling and forecast of climate variability and change, agricultural productivity, water resources management, drought prediction, flood area mapping, and ecosystem health monitoring all require information on the status of soil moisture. The outcomes from these applications all have direct impacts on the global environment and human society. Measuring surface soil

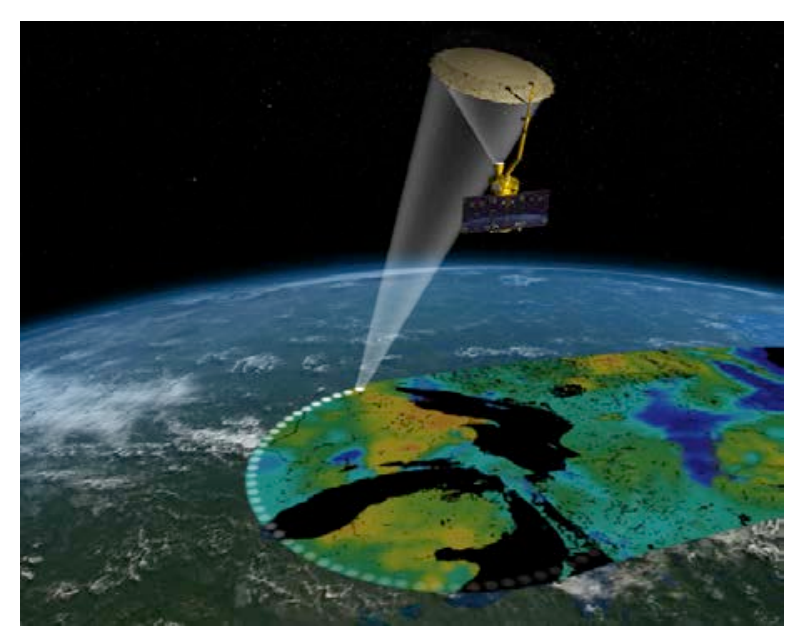

Figure 1. SMAP's conical scanning instrument produces a swath width of $1000 \mathrm{~km}$ to enable global coverage in 3 days.

moisture with the required accuracy and resolution (spatial and temporal) is imperative to fulfill the needs of these and other applications.

The NASA Soil Moisture Active Passive (SMAP) Mission was recommended as the one of the tier 1 missions in the National Research Council's (NRC) Decadal Survey Report, Earth Science and Applications from Space: National Imperatives for the Next Decade and Beyond [1]. The key science requirements for the SMAP mission are to provide accurate soil moisture and freeze/thaw classification for hydrometeorology, hydroclimatology and carbon cycle studies.

SMAP mission uses an L-band radar and an L-band radiometer for concurrent, coincident measurements integrated as a single observation system [2]. The radiometer and radar share one common antenna reflector, which is a 6-m mesh deployable antenna. The antenna design is based on the offset parabola reflector design with one antenna feed to produce the antenna beam pointing at an incidence angle of about 40 degrees on the earth surface 
(Fig. 1). The mesh antenna together with the feed is positioned on a spinning assembly with a conical scanning rate of about 14 rotations per minute. The resulting swath

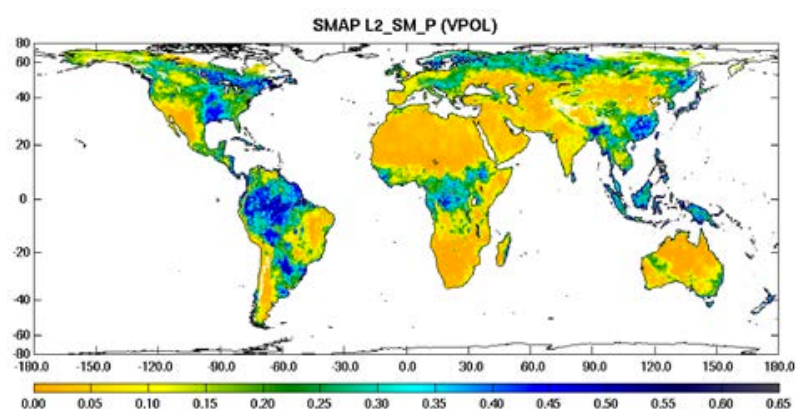

Figure 2. A three-day composite of the soil moisture derived from the vertically polarized brightness temperatures acquired by the SMAP radiometer during May 25-27, 2015.

width is about $1000 \mathrm{~km}$, which allows global coverage every 3 days.

The SMAP radiometer resolution is $\sim 40 \mathrm{~km}$, while the SMAP L-band SAR provided backscatter measurements at higher resolution ( 1 to $3 \mathrm{~km})$. The accuracy of the radar is limited for soil moisture sensing, however, by the higher sensitivity of radar to surface roughness and vegetation scattering. The significant advantage provided by SMAP is the concurrent L-band radar and radiometer measurement capability, whereby the radar and radiometer measurements could be effectively combined to derive soil moisture estimates with intermediate accuracy and resolution $(\sim 9 \mathrm{~km})$ that meet the SMAP science requirements.

\section{PRELIMINARY ASSESSMENT OF SCIENCE PERFORMANCE}

The SMAP instruments have been calibrated using the data from other satellites and external targets. The calibration of SMAP radiometer's noise diode was achieved using the ocean targets and cold sky. Cross-comparison with the European Space Agency's Soil Moisture and Ocean Salinity (SMOS) radiometer data over land and ocean surfaces indicates an excellent agreement of about $1 \mathrm{~K}$. The radiometric calibration of the radar was performed using the data from the Japanese Phased Array type Synthetic Aperture Radar (PALSAR). Independent assessment was performed by comparison with the Aquarius radar model function for ocean surfaces and indicated an agreement of about $0.5 \mathrm{~dB}$ for all polarizations. The calibrated L1 radar data have been released through the Alaska Satellite Facility (ASF) and the radiometer data through the National Snow and Ice Data Center (NSIDC).

The excellent calibration of radiometer and radar data has allowed the production and early release of L2/3/4 soil moisture and L3 freeze/thaw beta-level products through NSIDC. Figure 2 provides a global image of the soil moisture derived from the radiometer data on a $36 \mathrm{~km}$ grid.

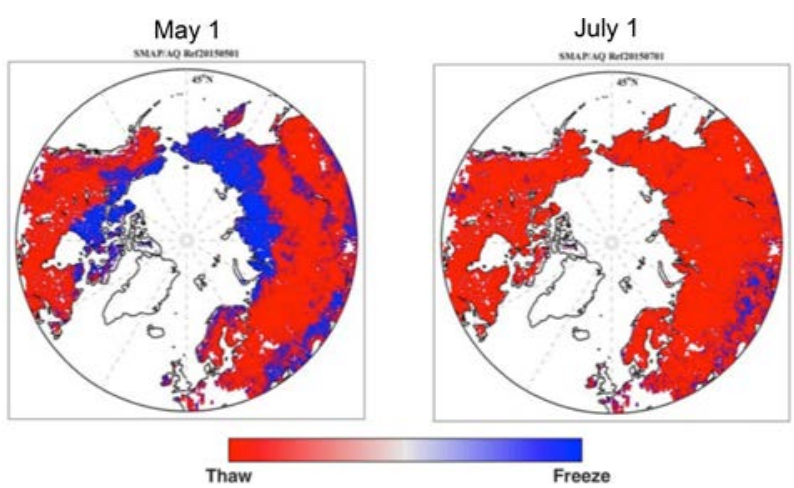

Figure 3. SMAP freeze/thaw maps on May 1 and July 1, 2015. The radar data for reference states were derived from a hybrid of SMAP and Aquarius data.

The data depict dry soil moisture surfaces in the western United States, Sahara, Middle East and central Australia. Wetter soil due to precipitation is evident in the Southern United States, the pampas regions in South America, Congo River basin and South East Asia. The 9-km soil moisture products derived from the disaggregated brightness temperatures using active radar data have revealed the same features.

The freeze/thaw products were also generated using the radar data at a spatial resolution of $3 \mathrm{~km}$. The algorithm is based on the threshold of the data normalized by the minimum level of radar signals in winter and maximum in summer. Figure 3 illustrates the freeze/thaw maps on May 1 and July 1 . The change from May to July is clearly within the expectation.

\section{CALIBRATION AND VALIDATION}

The SMAP project has been conducting a calibration/validation (Cal/Val) program to assess the quality of soil moisture and freeze/thaw products. The $\mathrm{Cal} /$ Val program includes the comparison of data with many core $\mathrm{Cal} / \mathrm{Val}$ sites, where spatially distributed in situ sensors were deployed within the reference grids, data comparison with sparse in-situ networks, inter-satellite comparison, and field campaigns.

The preliminary $\mathrm{Cal} / \mathrm{Val}$ assessment has indicated excellent quality of the soil moisture and freeze/thaw products. The key findings include 1) the unbiased root mean square error (ubRMSE) of the passive soil moisture product has met the 4 percent volumetric accuracy requirement [3], 2) soil moisture retrieval bias for some $\mathrm{Cal} / \mathrm{Val}$ sites or land cover types remains to be reduced, and 
3) the detection of freeze/thaw states has well exceeded the requirement of 80 percent classification accuracy.

Figure 4 illustrates a comparison of the passive soil moisture product with the in situ data acquired from the Texas Soil Moisture Observation Network (TxSON) Cal/Val

\section{SCIENCE RECOVERY}

One of SMAP's key science requirements is to produce soil moisture products at a spatial resolution of better than $10 \mathrm{~km}$ for hydrometeorology applications. This was to be achieved

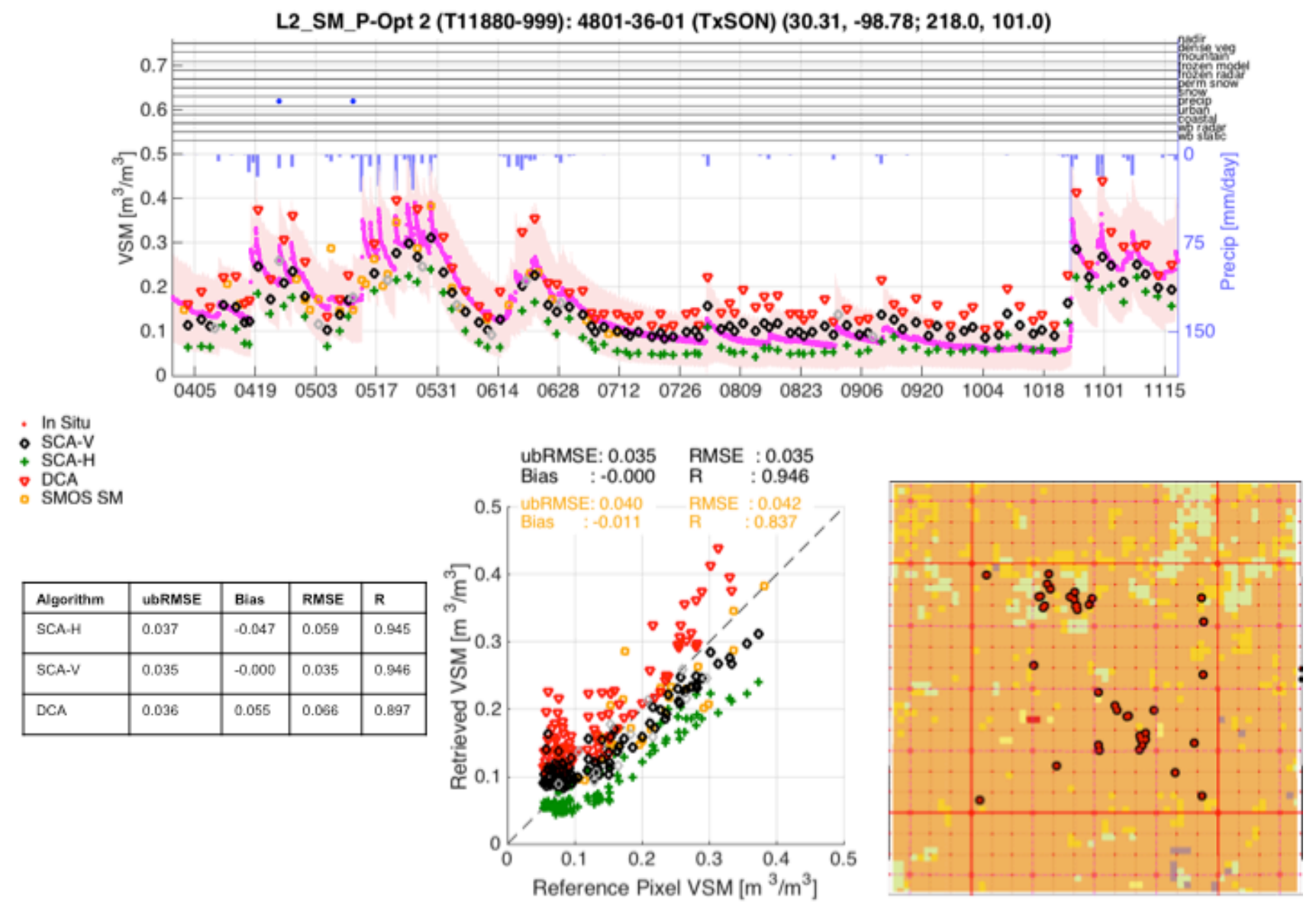

Figure 4. Comparison with the in situ data from the TxSON Cal/Val site. The upper panel illustrates the time series comparison. The bottom right indicates the locations of in situ sensors in the $36 \mathrm{~km}$ grid. The bottom middle indicates the scatter plot of satellite retrieved volumetric soil moisture (VSM) and reference (in situ) VSM. The statistics of differences is provided in the bottom left panel for Single Channel Algorithm H-pol (SCA-H), Single Channel V-pol (SCA-V) and Dual Channel Algorithm (DCA). Soil moisture results from SMOS are also shown.

site. The time series comparison clearly indicates the changes of soil moisture due to precipitation events and subsequent dry downs. All three algorithms produce very similar ubRMSE and correlation. The bias is smaller for SCA-V, leading to a smaller RMSE than the other two algorithms for the TxSON site. However, the SCA-H has a better performance for some other sites. After we average the statistics using the data from all core Cal/Val sites, SCA$\mathrm{V}$ has a slightly better performance overall for the beta-level products, and is consequently selected as the current baseline product. by using high-resolution radar data to disaggregate brightness temperatures on $9 \mathrm{~km}$ resolution grids. Unfortunately the SMAP radar transmitter ceased operation on July 7, 2015. Since then the SMAP project has been conducting two tasks to enhance the resolution of radiometer data products. One of the tasks will take advantage of the oversampling characteristics of the SMAP radiometer data along scan and along track. Two resolution enhancement techniques are being considered, including the Backus Gilbert (BG) optimum interpolation [4] and de-convolution $[5,6]$. The other task will match up the SMAP radiometer 
data and Sentinel-1's C-band SAR data, and will then apply the SMAP Active-Passive (AP) algorithm to produce the higher resolution soil moisture products. These two tasks will produce complementary products; it is expected that the BG or deconvolution techniques will lead to a global product with a spatial resolution of about 20 to $30 \mathrm{~km}$ resolution, while the SMAP/Sentinel-1 AP algorithm will produce soil moisture with resolution as high as $1 \mathrm{~km}$, but only for regions where the Sentinel-1 data are available.

Figure 5 provides an illustration of the BG interpolation
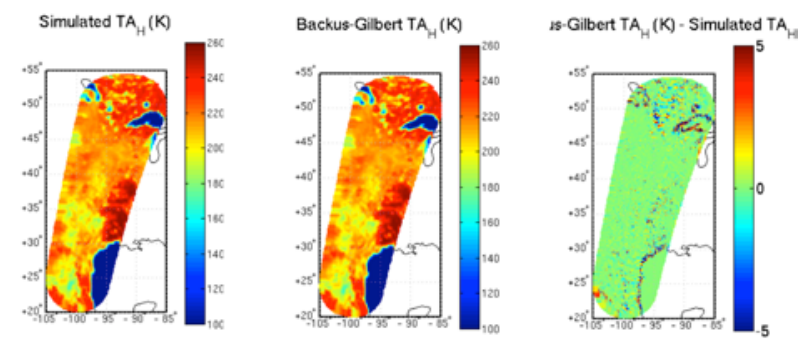

Figure 5. Illustration of the Backus-Gilbert interpolation algorithm applied to simulated SMAP radiometer data. Left panel simulated data; middle panel: BG output; right panel: difference between simulation and BG interpolation.

algorithm applied to a half orbit of simulated SMAP radiometer brightness temperatures. The interpolation was carried out on $3 \mathrm{~km}$ grids aligned with the along-track and across-track coordinate system. The interpolated data will then be averaged to produce data on the Equal-Area Scalable-2 Earth Grid (EASE-2) at 9 or $18 \mathrm{~km}$ resolution.

The potential of the AP soil moisture algorithm for combined SMAP radiometer/Sentinel-1 radar data is indicated in Fig. 6. The Sentinel-1 SAR data was used to disaggregate the SMAP radiometer data at a spatial resolution of $1 \mathrm{~km}$ for a site in Southern Canada. The highresolution features are clearly visible in the soil moisture image although the algorithm parameters require optimization. We will be applying the SMAP Cal/Val program to the SMAP/Sentinel-1 AP soil moisture product, and will be conducting the SMAPVEX campaign in 2016 for direct validation of the disaggregated brightness temperature data.

\section{APPLICAITONS}

In spite of the loss of SMAP radar in July, the SMAP radiometer has been performing very well. The radiometer soil moisture products have been used to monitor the severe flooding caused by heavy rainfall in Texas in May-June and in South Carolina in early October. More recent flooding events in Missouri and South America in December were also indicated in the SMAP soil moisture products.
The calibrated radiometer data have also generated several new science products, including the vegetation opacity, ocean surface salinity, and extreme ocean surface wind speed for hurricanes. The SMAP vegetation opacity agreed qualitatively well with the land cover map and independent LIDAR vegetation height measurement. The change of vegetation opacity has a good correlation with the crop growth and harvest periods in South Fork, Iowa. The SMAP radiometer has been operating over ocean surfaces as

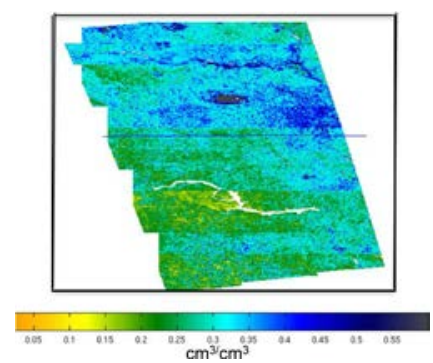

Figure 6. High resolution $(1 \mathrm{~km})$ soil moisture derived from combined SMAP radiometer and Sentinel-1 radar data in southern Canada.

well. The ocean data have been used to retrieve the sea surface salinity (SSS), which will become particularly critical after the loss of NASA Aquarius mission in June for ocean circulation and water cycle research.

\section{ACKNOWLEDGEMENT}

The work described in this paper was carried out by the Jet Propulsion Laboratory, California Institute of Technology under a contract with the National Aeronautics and Space Administration. The authors would like to acknowledge the contributions by the SMAP team members on the $\mathrm{Cal} / \mathrm{Val}$ effort and the figures presented in the article.

\section{REFERENCES}

[1] National Research Council, "Earth Science and Applications from Space: National Imperatives for the Next Decade and Beyond,” pp. 400, 2007.

[2] D. Entekhabi, E. G. Njoku, P. E. O’Neill, K. H. Kellogg, W. T. Crow, W. N. Edelstein, J. K. Entin, S. D. Goodman, T. J. Jackson, J. Johnson, J. Kimball, J. R. Piepmeier, R. D. Koster, N. Martin, K. C. McDonald, M. Moghaddam, S. Moran, R. Reichle, J. C. Shi, M. W. Spencer, S. W. Thurman, L. Tsang, and J. Van Zyl, The Soil Moisture Active Passive (SMAP) Mission, Proceedings of IEEE, Vol. 98,No. 5,pp. 704-716, May 2010.

[3] Jackson, T., P. O’Neill, E. Njoku, S, Chan, R. Bindlish, A. Colliander, F. Chen, M. Burgin, S. Dunbar, J. Piepmeier, S. Yueh, D. Entekhabi, M. Cosh, T. Caldwell, J. Walker, X. Wu, A. Berg, T. Rowlandson, A. Pacheco, H. McNairn, M. Thibeault, J. Martínez-Fernández, Á. González-Zamora, M. Seyfried, D. Bosch, 
P. Starks, D. Goodrich, J. Prueger, M. Palecki, E. Small, J. Calvet, W. Crow, and Y. Kerr, November 16, 2015. Calibration and Validation for the L2/3_SM_P Beta-Release Data Products, Version 2, SMAP Project, JPL D-93981, Jet Propulsion Laboratory, Pasadena, CA.

[4] G. Poe, "Optimum Interpolation of Imaging microwave Radiometer Data,” IEEE Trans. Geosci. Remote Sens., Vol. 28, No. 5, pp. 800-810, September 1990.

[5] D. G. Long and D. L. Daum, "Spatial Resolution Enhancement of SSM/I Data,” IEEE Trans. Geosci. Remote Sens., Vol. 36, No. 2, pp. 407-417, September 1998.

[6] I. Yanovsky, A. Tanner, and B. Lambrigtsen, "Efficient Deconvolution and Spatial Resolution Enhancement From Continuous and Oversampled Observations In Microwave Imagery, MicroRad Conference, March 2014, Pasadena. 